\title{
Stability analysis in spanwise-periodic double-sided lid-driven cavity flows with complex cross-sectional profiles
}

\author{
Javier de Vicente*, Daniel Rodríguez, Vassilis Theofilis, Eusebio Valero \\ School of Aeronautics, Universidad Politécnica de Madrid, Pza. Cardenal Cisneros 3, E-28040 Madrid, Spain
}

\section{A R T I C L E I N F O}

Keywords:

Spectral multi-domain method

Chebyshev collocation technique

BiGlobal instability analysis

Lid-driven cavity flows

Sparse-serial vs dense-parallel algorithm

\begin{abstract}
A B S T R A C T
Three-dimensional linear instability analyses are presented of steady two-dimensional laminar flows in the lid-driven cavity defined by [15] and further analyzed in the present volume [1], as well as in a derivative of the same geometry. It is shown that in both of the geometries considered three-dimensional BiGlobal instability leads to deviation of the flow from the two-dimensional solution; the analysis results are used to define low- and high-Reynolds number solutions by reference to the flow physics. Critical conditions for linear global instability and neutral loops are presented in both geometries.
\end{abstract}

\section{Introduction}

Lid-driven cavity (LDC) flows have been a mainstay of fluid dynamics research for decades; see [28] for a review. By far the most studied configuration is that of two-dimensional flow set up by constant speed motion of a flat surface over a square or rectangular container. Following the first numerical solutions of the LDC problem by Burggraf [5], from a physical point of view the existence of steady two-dimensional incompressible flow at low Reynolds numbers in rectangular domains has been established experimentally in the flow visualizations of Pan and Acrivos [23]. From a numerical point of view, primarily owing to the richness of physical phenomena associated with the vortical structures set up both in the main part [4] and the corners of the domain [20], two-dimensional LDC flows have served as benchmark configurations for incompressible Navier-Stokes solvers; the citation map of $[14,27]$ testifies to this effect. As algorithms for the solution of the incompressible equations of motion became more sophisticated, the group of Michel Deville having made substantial contributions to this effect in the context of spectral methods $[10,26]$, steady flow solutions in twodimensional cavity geometries of ever-increasing cross-sectional complexity started appearing in the literature [22,33,21].

Vivid discussions of "high-Reynolds number", numerically-obtained two-dimensional LDC flows in a square domain may still be found in the literature [13], although it is well-understood for a decade now that such flows loose their stability due to linear amplification of three-dimensional global eigenmodes [31,32]. In

\footnotetext{
* Corresponding author. Tel.: +34 913366326.

E-mail address: javierdvb@gmail.com (J. de Vicente).
}

square LDC flow the first reliable global instability analysis results were presented by Ding and Kawahara [12], but the full map of unstable modes, including a stationary critical three-dimensional instability which becomes unstable at lower Reynolds numbers than the mode discovered by [12], was first provided independently by Theofilis [30] and Albensoeder et al. [2]. Consequently, in a two-dimensional square LDC flow context it is precisely the linear critical Reynolds number for three-dimensional linear instability, $R e_{\text {crit,3d }} \approx 782$ [30], which defines a threshold between what may be considered as low and high Reynolds number: below $R e_{\text {crit,3d }}$ physically realizable two-dimensional laminar LDC flows do exist [23], while numerical solutions of the Navier-Stokes equations of motion at any $R e \geqslant R e_{\text {crit,3d }}$ cannot be realized experimentally, since they are three-dimensionally unstable and, as such, they may be considered as unphysical.

The present contribution examines global instability of two LDC flows with relatively complex cross-sectional profiles. The first geometry is that proposed by Hinatsu and Ferziger [15], and further analyzed in detail by Zhou et al. [33], as benchmark for algorithms capable of coping with geometric discontinuities in numerical solutions of the incompressible Navier-Stokes equations, while the second cross-sectional profile is a symmetrized version of the first geometry. In both cases, accurate steady laminar two-dimensional solutions are obtained by direct numerical simulation, employing spectral collocation and spectral multidomain $[10,26]$. Subsequently, these solutions are analyzed with respect to their three-dimensional global linear instability using two independent solutions of the BiGlobal eigenvalue problem, both of which use the same spectral-multi-domain algorithms and either dense [24] or sparse [8,7] linear algebra for the recovery 

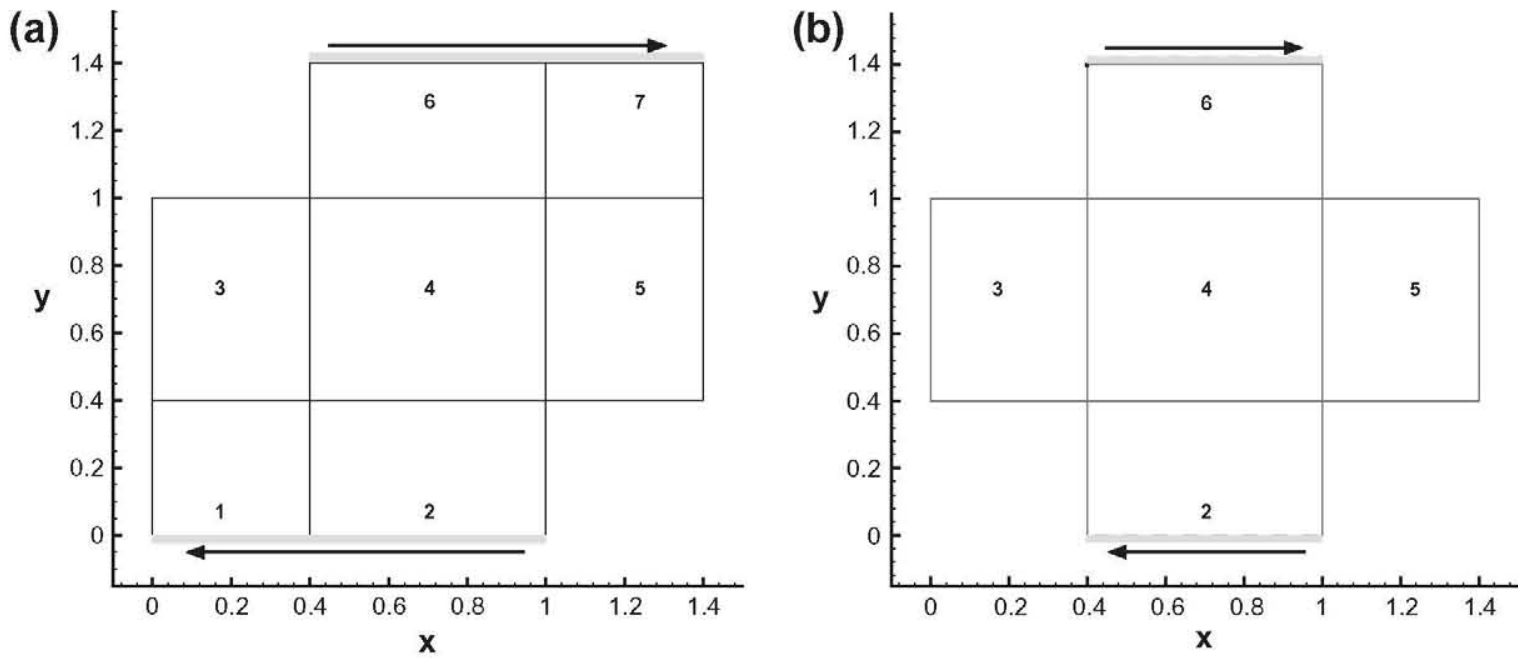

Fig. 1. Domain decomposition of the lid-driven cavity geometries analyzed. (a) Hinatsu and Ferziger [15]; (b) Same geometry, less subdomains 1 and 7.

of the eigenspectrum. Critical conditions and neutral loops are established for both geometries examined, thus defining in both configurations the boundaries between physically realizable and purely numerical two-dimensional steady solutions of the equations of motion. Fig. 1 presents the geometries studied, while Section 2 introduces the theoretical concepts employed and elements of the numerical algorithms utilized. Results and their implications are presented in Section 3 and a short discussion in Section 4 closes the presentation.

\section{Theory and numerical algorithms}

\subsection{Basic flow calculation}

BiGlobal instability analysis requires a steady two-dimensional basic state upon which unsteady small-amplitude disturbances are superimposed. This basic flow solution has been computed by integration of the two-dimensional incompressible laminar NavierStokes and continuity equations. In non-dimensional form, in the absence of volumetric forces, the equations of motion are written as

$\frac{\partial \overline{\mathbf{v}}}{\partial t}+(\overline{\mathbf{v}} \cdot \nabla) \overline{\mathbf{v}}=\frac{1}{\operatorname{Re}} \nabla^{2} \overline{\mathbf{v}}-\nabla \bar{p}$,

$\nabla \cdot \overline{\mathbf{v}}=0$.

Here, $\overline{\mathbf{v}}=(\bar{u}, \bar{v})^{T}$, and $\bar{p}$ denote the velocity component vector and pressure, respectively, while all variables are non-dimensionalized by a characteristic cavity dimension velocity, Re being the Reynolds number.

Chebyshev spectral collocation is applied for the spatial discretization. To handle the nontrivial geometries appearing, the multidomain techniques originally presented by Demaret and Deville $[9,10]$ is employed. For the time discretization a semi-implicit coupled scheme is used, incorporating artificial compressibility $[6,29,11]$. Although transient solutions recovered by this method may not be as accurate as those obtained by alternative approaches, the present focus is on recovering steady states, for which the algorithm was found to perform adequately, as shown later. Nonetheless, following some experimentation, quite acceptable transient solutions have also been obtained [7]. The discretization used was based on the semi-implicit system

$\frac{1}{d t} \overline{\mathbf{v}}^{n+1}-\frac{1}{\operatorname{Re}} \nabla^{2} \overline{\mathbf{v}}^{n+1}+\nabla \bar{p}^{n+1},=\frac{1}{d t} \overline{\mathbf{v}}^{n}-\left(\overline{\mathbf{v}}^{n} \cdot \nabla\right) \overline{\mathbf{v}}^{n}$,

$\frac{\epsilon}{d t} \bar{p}^{n+1}+\nabla \cdot \overline{\mathbf{v}}^{n+1}=\frac{\epsilon}{d t} \bar{p}^{n}$, which treats convective terms in the Navier-Stokes equation explicitly, while pressure and viscous terms are treated implicitly. The choice of an Euler method for time advancing is due to its simple implementation and its good stability properties when a steady solution is sought. The convergence criterion utilized is

$\left|\frac{\overline{\mathbf{v}}^{n+1}-\overline{\mathbf{v}}^{n}}{\overline{\mathbf{v}}^{n}}\right| \leqslant 10^{-14}$.

Using this methodology the computation may be reduced to the solution of linear systems in which the explicit terms are updated at each time step. The main advantage of this matrix interpretation is that boundary conditions are imposed prior to the LU decomposition, such that the matrix factorization is calculated once and stored, in order to be used repeatedly during the time-marching.

In the cavity geometries monitored, the singular nature of the boundary conditions at corners with velocity discontinuities can destroy the overall spectral accuracy. In order to avoid this issue, the moving lid motion has been regularized using the procedure suggested by Leriche et al. [18]

$\bar{u}= \pm\left[1-\left(\frac{2 x-\left(x_{1}+x_{2}\right)}{x_{1}-x_{2}}\right)^{18}\right]^{2}$

where $x_{1}$ and $x_{2}$ are the streamwise coordinates of the edges of the moving lids in the cavities considered. The maximum of the velocity defined in Eq. (6) and the length scale shown in Fig. 1 define the Reynolds number of the problem.

\subsection{BiGlobal instability analysis}

Let $\mathbf{q}(x, y, z, t)=(\mathbf{v}, p)^{T}$ be the variables vector in three-dimensional incompressible flow. The flow is decomposed into the laminar steady two-dimensional basic flow, $\overline{\mathbf{q}}=\langle\overline{\mathbf{v}}, \bar{p})^{T}$, calculated previously, with small-amplitude three-dimensional modal disturbances superposed, according to

$\mathbf{q}=\overline{\mathbf{q}}+\varepsilon \hat{\mathbf{q}} \exp (\mathrm{i} \beta z+\omega t)+$ c.c.

Considering $\varepsilon \ll 1$, the continuity and Navier-Stokes equations are linearized around $\overline{\mathbf{q}}$, resulting in the generalized EVP

$\nabla \cdot \hat{\mathbf{v}}=0$

$(\overline{\mathbf{v}} \cdot \nabla) \hat{\mathbf{v}}+(\hat{\mathbf{v}} \cdot \nabla) \overline{\mathbf{v}}+\nabla \hat{p}-\frac{1}{R e} \nabla^{2} \hat{\mathbf{v}}=\omega \hat{\mathbf{v}}$.

The eigenvalues $\omega=\omega_{r}+i \omega_{i}$ describe the temporal behaviour of the eigenmodes: $\omega_{i}$ is a circular frequency, while $\omega_{r}$ is the growth rate. If eigenmodes are identified having $\omega_{r}>0$, the flow is 


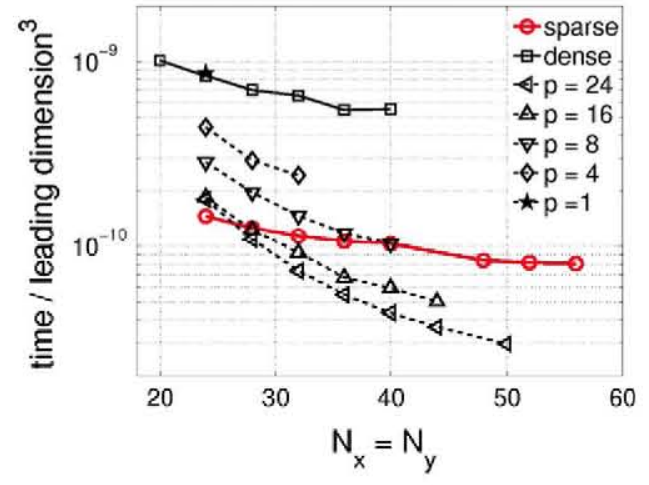

Fig. 2. CPU-time scaled with $L D^{3}$ (following Eq. (11)), required for the computation of the spectrum using sparse serial code (open round symbol) and several configurations of the parallel dense code on $p$ processors.

unstable and the disturbance in question will grow exponentially in time until saturated by non-linear effects. In other words, linear amplification of a single eigenmode, for any set of parameters $(R e, \beta)$ suffices for the flow to deviate from the original steady laminar basic state, $\overline{\mathbf{q}}$.

\subsection{Numerical solution of the eigenvalue problem}

Spectral collocation methods have been used for the discretization of the linear operator (8), (9), resulting in the matrix eigenvalue problem

$\mathbf{A X}=\omega \mathbf{B X}$.

A shift-and-invert implementation of the Arnoldi algorithm $[3,25]$ is used, in which only the matrix $\overline{\mathbf{A}}=(\mathbf{A}-\sigma \mathbf{B})^{-1} \mathbf{B}, \sigma$ being the shiftparameter, need be stored.

Regarding the computing requirements of BiGlobal instability analysis, sufficient resolution for the reliable recovery of the (Reynolds-number-dependent) fine eigenvector structure is only one of the related considerations. In addition, the multi-domain spectral collocation technique used in this work generates matrices of a particular sparsity pattern. Concretely, there exist two sources of sparsity in each subdomain, one associated with the spatial differential operator itself, i.e. the left-hand-side of Eqs. (8) and (9), and

Table 1

Comparisons of present basic flow results with those of Zhou et al. [33] and Nithariasu and Liu[21] at $R e=100$.

\begin{tabular}{lll}
\hline Primary vortex 1 & & \\
& $(x, y)$ & $\zeta(x, y)$ \\
Zhou et al. [33] & $(1.0172,1.1091)$ & -2.72390 \\
Nithiarasu and Liu [21] & $(1.0114,1.1035)$ & -2.69993 \\
Present results & $(1.0138,1.1091)$ & -2.73921 \\
Primary vortex 2 & & \\
& $(x, y)$ & $\zeta(x, y)$ \\
[33] & $(0.3828,0.2889)$ & -2.72310 \\
[21] & $(0.3865,0.2894)$ & -2.73231 \\
Present results & $(0.3861,0.2908)$ & -2.73906 \\
First secondary vortex 1 & & $\zeta(x, y)$ \\
& $(x, y)$ & 0.04399 \\
[33] & $(1.3556,0.4486)$ & 0.03975 \\
[21] & $(1.3566,0.4446)$ & 0.03606 \\
Present results & $(1.3584,0.4458)$ & \\
First secondary vortex 2 & & $\zeta(x, y)$ \\
& $(x, y)$ & 0.04401 \\
[33] & $(0.0444,0.9514)$ & 0.03695 \\
[21] & $(0.0424,0.9569)$ & 0.03606 \\
Present results & $(0.0415,0.9541)$ & \\
\hline
\end{tabular}

Table 2

Comparisons of present basic flow results with those of Zhou et al. [33] and Nithariasu and Liu [21] at $R e=400$.

\begin{tabular}{lll} 
Primary vortex & $(x, y)$ & \\
Zhou et al. [33] & $(0.7000,0.7000)$ & -1.54842 \\
Nithiarasu and Liu [21] & $(0.6995,0.6966)$ & -1.60552 \\
Present results & $(0.7000,0.7000)$ & -1.57392 \\
First secondary vortex 1 & & \\
& $(x, y)$ & $\zeta(x, y)$ \\
[33] & $(1.3500,0.4656)$ & 0.15569 \\
[21] & $(1.3483,0.4688)$ & 0.19767 \\
Present results & $(1.3472,0.4638)$ & 0.18102 \\
First secondary vortex 2 & & \\
& $(x, y)$ & $\zeta(x, y)$ \\
[33] & $(0.0500,0.9344)$ & 0.15777 \\
[21] & $(0.0569,0.9400)$ & 0.19385 \\
Present results & $(0.0523,0.9363)$ & 0.18103 \\
Second secondary vortex 1 & & $\zeta(x, y)$ \\
& $(x, y)$ & 1.38495 \\
[33] & $(0.4703,1.1625)$ & 1.70971 \\
[21] & $(0.4772,1.1610)$ & 1.50132 \\
Present results & $(0.4747,1.1671)$ & \\
Second secondary vortex 2 & & $\zeta(x, y)$ \\
[33] & $(x, y)$ & 1.38140 \\
[21] & $(0.9219,0.2375)$ & 1.59442 \\
Present results & $(0.9232,0.2385)$ & \\
\hline
\end{tabular}

another which is related to the sparsity pattern of the spectral collocation differentiation matrices themselves. However, the major source of sparsity in the problem at hand is due to the multi-domain implementation. The reason is that the leading dimension, $L D$, of the multi-domain matrices $\mathbf{A}, \mathbf{B}$ and $\overline{\mathbf{A}}$ scales as the product of the number of subdomains employed, $N_{d}$ times the number of collocation points per domain, $N_{p}$. Closer inspection reveals that in a multi-domain context $N_{d}$ subdomains are devoted to the discretization of the linear operator, while $N_{d}^{2}-N_{d}$ domains are practically zeroes, except for the entries dedicated to implementation of the interface condition between domains. In the latter context, enforcement of continuity across subdomains implies the substitution of a given number of discretized equations in favor of (discrete) interface conditions. The number of interface conditions depends on the order of the differential equation collocated in each subdomain; $C^{1}$ continuity is enforced at the interface when solving the second-order BiGlobal EVP. Clearly, the ratio between lines in the discrete matrix that are devoted to the implementation of interface conditions over that reserved for the solution of the equation itself must be kept low. For a given discretization this condition is violated if an increasingly large number of subdomains is considered. Conversely, this condition can always be satisfied by increasing resolution, for any given number of subdomains.

The block-diagonal structure of the matrix (when no interface conditions are considered) becomes the dominant consideration in choosing numerical methods for the linear algebra operations (matrix factorization, matrix-vector multiplication), since the number of non-zero elements of the matrix decreases rapidly as the number of subdomains increases. At this point one is faced with a dichotomy between a sparse or a dense approach to be followed for the linear algebra operations. Both have been employed in the present problem; a brief account of each methodology will be detailed hereafter.

\subsubsection{Dense parallel code}

Use of dense linear algebra is the most straightforward approach for the formation and operation on the matrices involved. However, treating $O\left(N_{d}^{2}\right)$ domains as dense, although only $O\left(N_{d}\right)$ subdomains carry information comes with a severe memory 
penalty. The use of distributed memory supercomputers was adopted as one solution for this issue, and a parallel version of the EVP solver was developed using MPI communications and the linear algebra libraries PBLAS and ScaLAPACK. Distributed storage and operation of the data implies that the maximum spatial resolution that can be used is only a function of the number of processors available $(p)$. In addition, parallelization drastically reduces the CPU-time. The most time-consuming task is to perform a LU

Table 3

Vorticity values and corresponding locations of the centres of primary and secondary vortices in the cross-cavity.

\begin{tabular}{llll}
\hline$R e$ & & $(x, y)$ & $\zeta(x, y)$ \\
\hline 200 & Primary & $(0.7707,1.2326)$ & -5.03919 \\
& Vortices & $(0.6293,0.1673)$ & -5.04023 \\
& Secondary & $(0.5164,0.6778)$ & 0.33976 \\
\multirow{2}{*}{500} & Vortices & $(0.8836,0.7221)$ & 0.33968 \\
& Primary & $(0.7431,1.1876)$ & -4.27435 \\
& Vortices & $(0.6569,0.2122)$ & -4.27317 \\
1000 & Sec. Vort. & $(0.7,0.7)$ & 0.67221 \\
& Primary & $(0.7255,0.1721)$ & -3.93792 \\
& Vortices & $(0.6745,0.2278)$ & -3.93796 \\
& Secondary & $(0.4701,0.7322)$ & 0.73701 \\
& Vortices & $(0.9299,0.6676)$ & 0.73707 \\
\hline
\end{tabular}
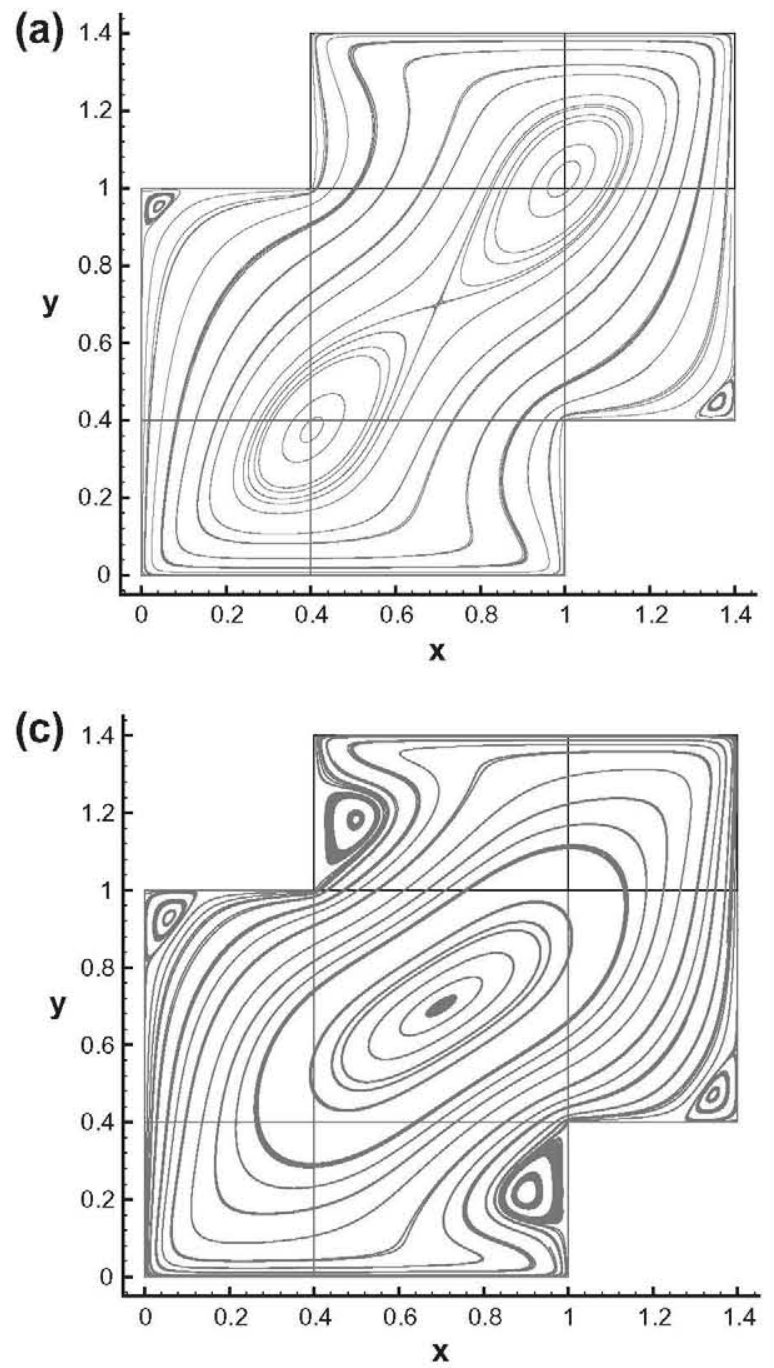

decomposition of the distributed matrix $\mathbf{A}$. This operation requires $\mathcal{O}\left(L D^{3}\right)$ operations, while the work load is balanced among the used processors:

$C P U-$ time $\sim \frac{L D^{3}}{p}$.

The scalability test performed on JUGENE (http://www.fz-juelich.de/jsc/jugene) show that the CPU-time is reduced proportionally to the number of processors, in agreement with the ideal scaling, using up to 4.096 processors. The latter case corresponds to a problem in which the matrix $\mathbf{A}$ has leading dimension equal to 252.000 , and requires distributed storage for 1 Terabyte of memory, being until today the world-wide largest hydrodynamic EVP solved. More details can be found in [24].

\subsubsection{Sparse serial code}

The interface conditions briefly discussed earlier prohibit use of straightforward linear algebra techniques for block-diagonal matrices. However, the nearly block-diagonal structure of the matrices suggests making use of sparse techniques in order to improve the efficiency of the numerical EVP solution. Sparsity is exploited using the Compressed Column Format (CCF) in order to store only the non-zero entries of the matrix, as well as the associated locations. The CCF stores the sparse matrix in three vectors:
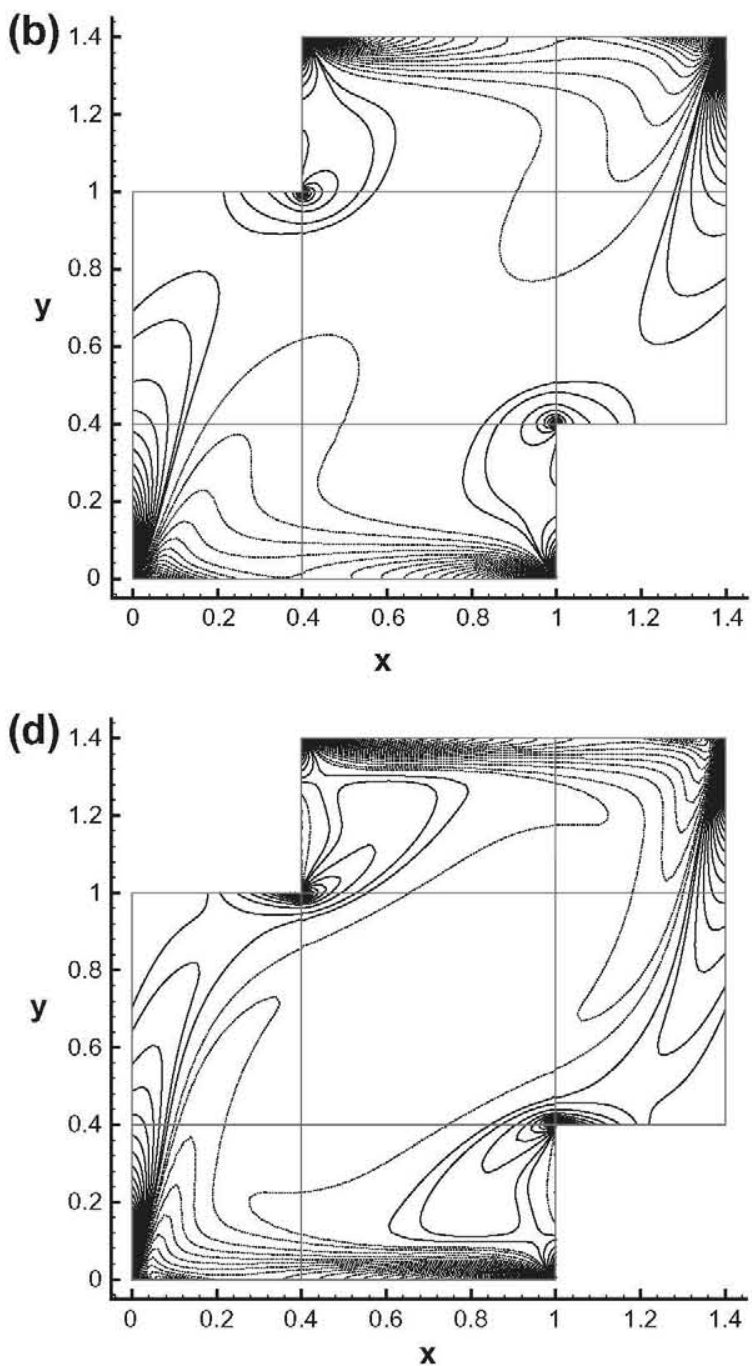

Fig. 3. Basic flow solutions in the geometry of Hinatsu and Ferziger [15]. (a) $\psi(x, y)$ at $\operatorname{Re}=150 ;(\mathrm{b}) \zeta(x, y)$ at $\operatorname{Re}=150 ;(\mathrm{c}) \psi(x, y)$ at $\operatorname{Re}=500 ;(\mathrm{d}) \zeta(x, y)$ at $\operatorname{Re}=500$. 
one for the non-zero values, another for the row location in each column of these non-zero elements and a third one for summing the number of entries in each column. Using this computing strategy the entries of the matrices discretizing the linear algebra problems arising in the two-dimensional DNS and the eigensystem
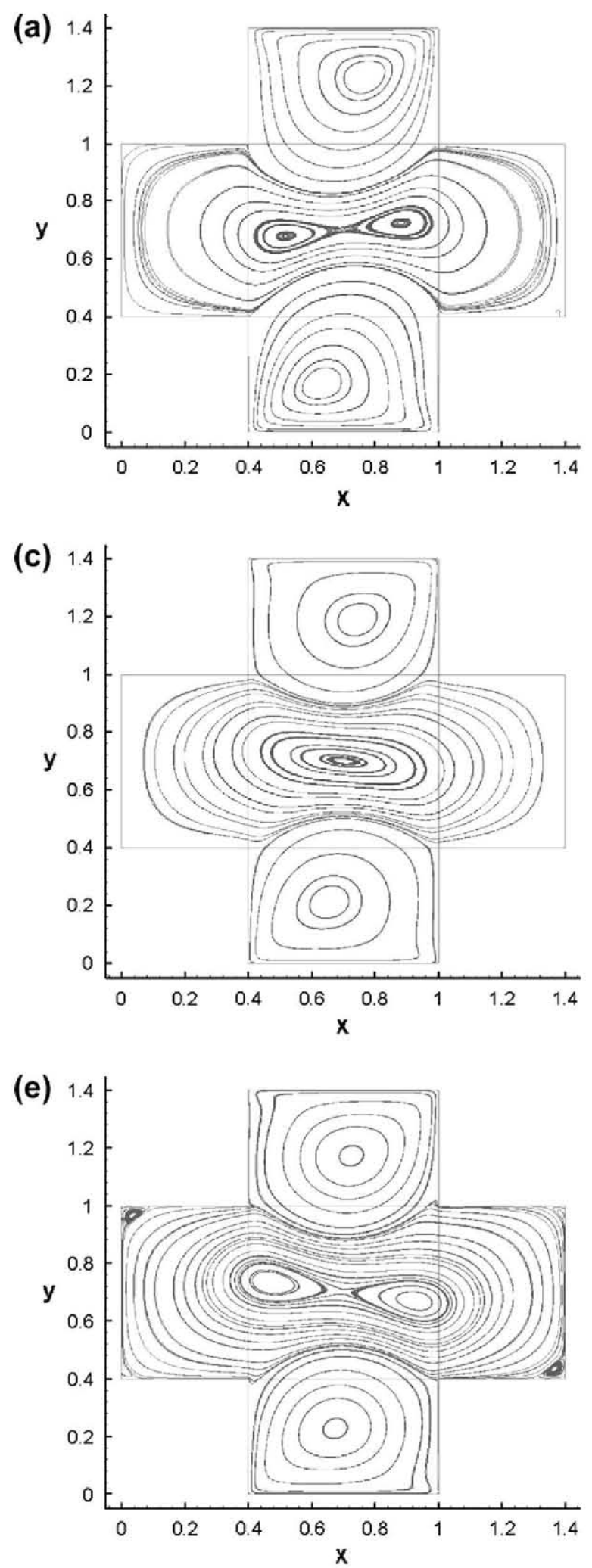

computation are formed explicitly, but the corresponding matrices are never stored as two-dimensional objects.

The LU-decompositions needed both in the context of the base flow calculation and the EVP solver, are performed using the SuperLU package [19], while the Arnoldi iterative method utilized
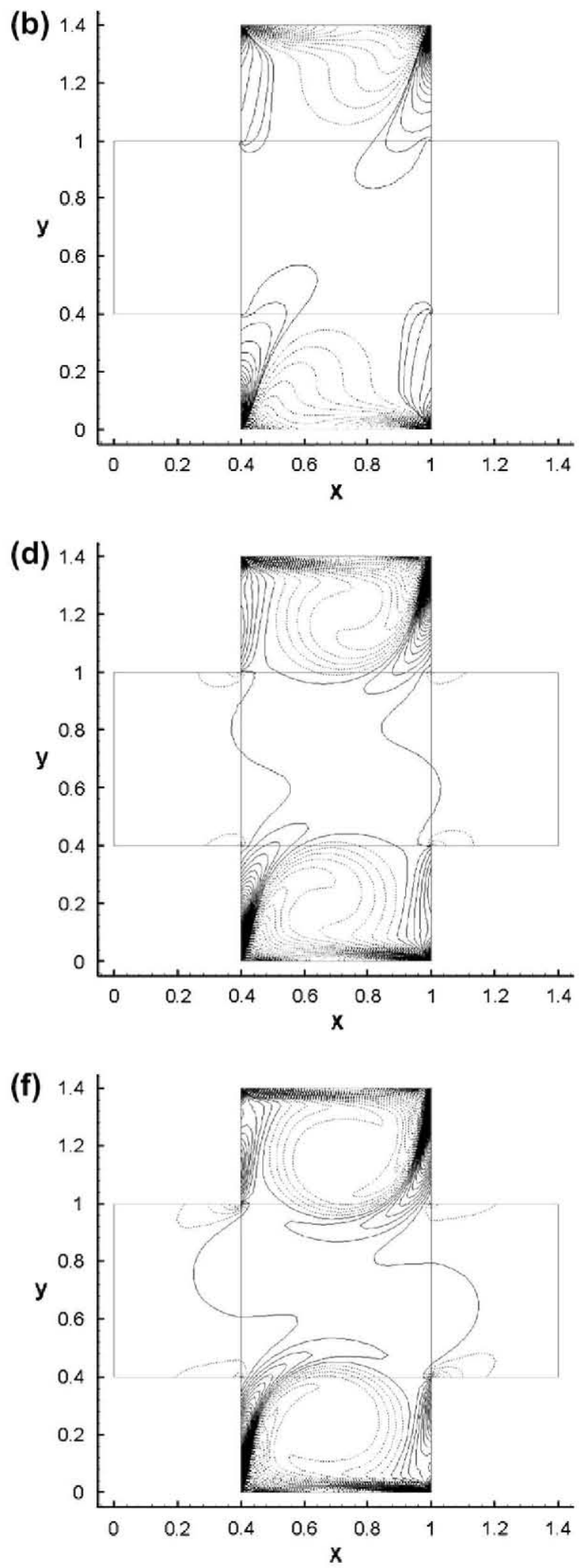

Fig. 4. Basic flow solutions in the cross geometry. (a) $\psi(x, y)$ at $R e=200$; (b) $\zeta(x, y)$ at $R e=200$; (c) $\psi(x, y)$ at $R e=500$; (d) $\zeta(x, y)$ at $R e=500$ (e) $\psi(x, y)$ at $R e=1000$ (f) $\zeta(x, y)$ at $R e=1000$. 
for the recovery of the leading Ritz values and vectors has been implemented using the ARPACK software package [17]. Both the SuperLU and ARPACK packages support the CCF sparse format and are reported to provide good serial and parallel performance; here, serial computing has been adopted.

\subsubsection{Comparisons of dense-vs. sparse eigenvalue problem solutions}

A detailed comparison of the relative performance of the serial sparse and parallel dense EVP solver has been made. Lack of space prohibits providing full details here; one aspect that is highlighted regards the identification of a threshold at which one numerical solution approach becomes more competitive over the other. Fig. 2 shows the appropriately scaled wall-clock time taken by the dense parallel approach to solve the eigenvalue problem (10) serially $(p=1)$ or on a number of up to $p=24$ processors, as a function of the number of collocation points per direction, $N_{x}=N_{y}$, used to discretize each of the components of the disturbance vector $\hat{\mathbf{q}}$ in

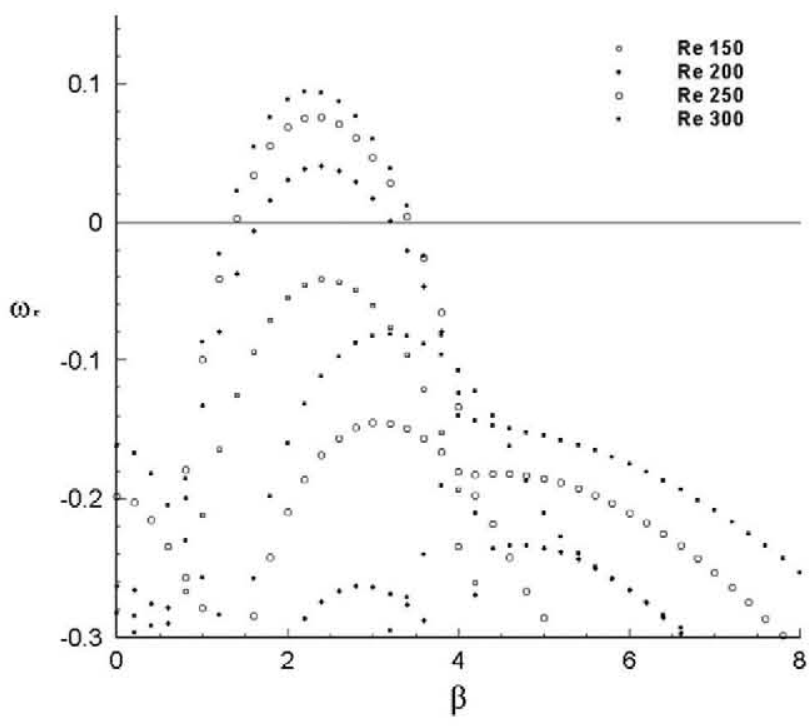

Fig. 5. Dependence of the amplification rate on the spanwise wavenumber parameter $\beta$ for a range of stable and unstable Reynolds numbers in the geometry defined by Hinatsu and Ferziger [15].

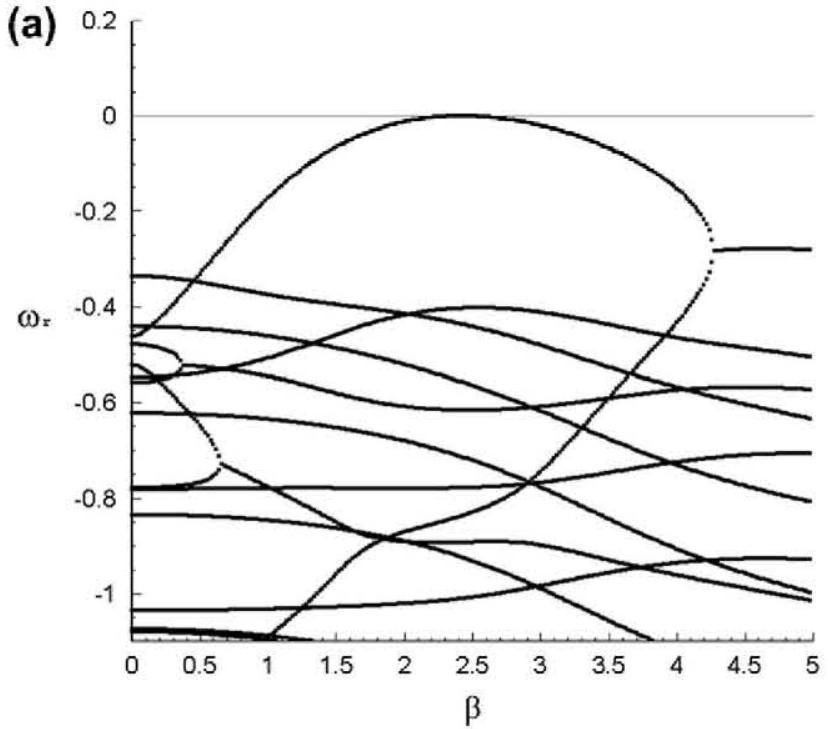

(7) in a single domain. A three-domain configuration has been used for these comparisons.

In the same figure, the CPU time taken by the sparse serial solver for the same problem is also shown. The latter is found to scale approximately as the cube of the leading dimension, as expected from the dense nature of the LU-decomposition. The parallel solver is also seen to scale in the same manner, although as the number of processors increases, a certain gain over the theoretical value is to be observed. On the other hand, as also expected from a theoretical point of view, the sparse solution is one order of magnitude more efficient than its dense counterpart, at all resolutions examined. Finally, for the problem at hand, it takes a matrix leading dimension upwards of $19,200=\left(N_{x}=40\right) \times\left(N_{y}=40\right) \times$ (4equations) $\times\left(N_{d}=3\right.$ domains $)$ and more than eight processors for the dense to become competitive with the sparse approach. It is worth remarking that the number of domains considered in this paper is higher than the $N_{d}=3$ domains considered in the test problem, and that the sparse code is increasingly more efficient as the number of subdomains is increased. Consequently, in all subsequent computations the sparse code has been employed for the solution of the generalized eigenvalue problem (10).

\section{Results}

Our first concern is with validation of the basic flow solution, $\overline{\mathbf{q}}$ in (7), since its accuracy conditions that of the subsequent instability analysis. Tables 1 and 2 present comparisons of the steady basic flow results of Zhou et al. [33] and Nithiarasu and Liu [21] alongside those of the present computations. Shown is the location of the primary and secondary vortices at $R e=100$ and 400 , as well as the value of the basic flow vorticity, $\zeta$, at those locations. The three computations deliver results within fractions of one percent of each other as far as the primary vortices are concerned, while the same level of agreement is also shown in the locations of the secondary vortices, but not on the values of the corresponding vorticity; the relative discrepancy in the latter quantity is found to be of the order of $10 \%$ in all three possible two-way comparisons.

The differences between the results of either of the earlier computations and the present work may be attributed to several factors. First, the temporal convergence criterion is substantially more stringent in ours compared with either of the earlier

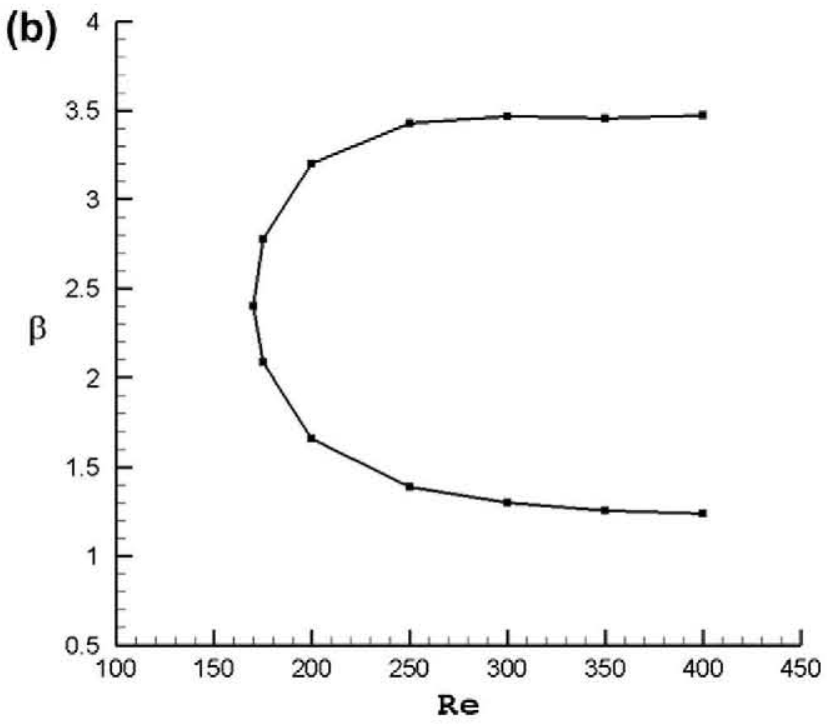

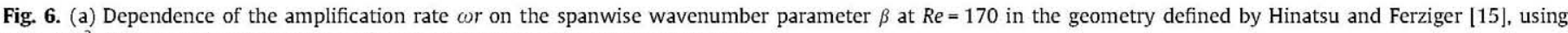
$\Delta \beta=10^{-3}$. (b): Neutral stability curve of the leading mode in the same geometry. 

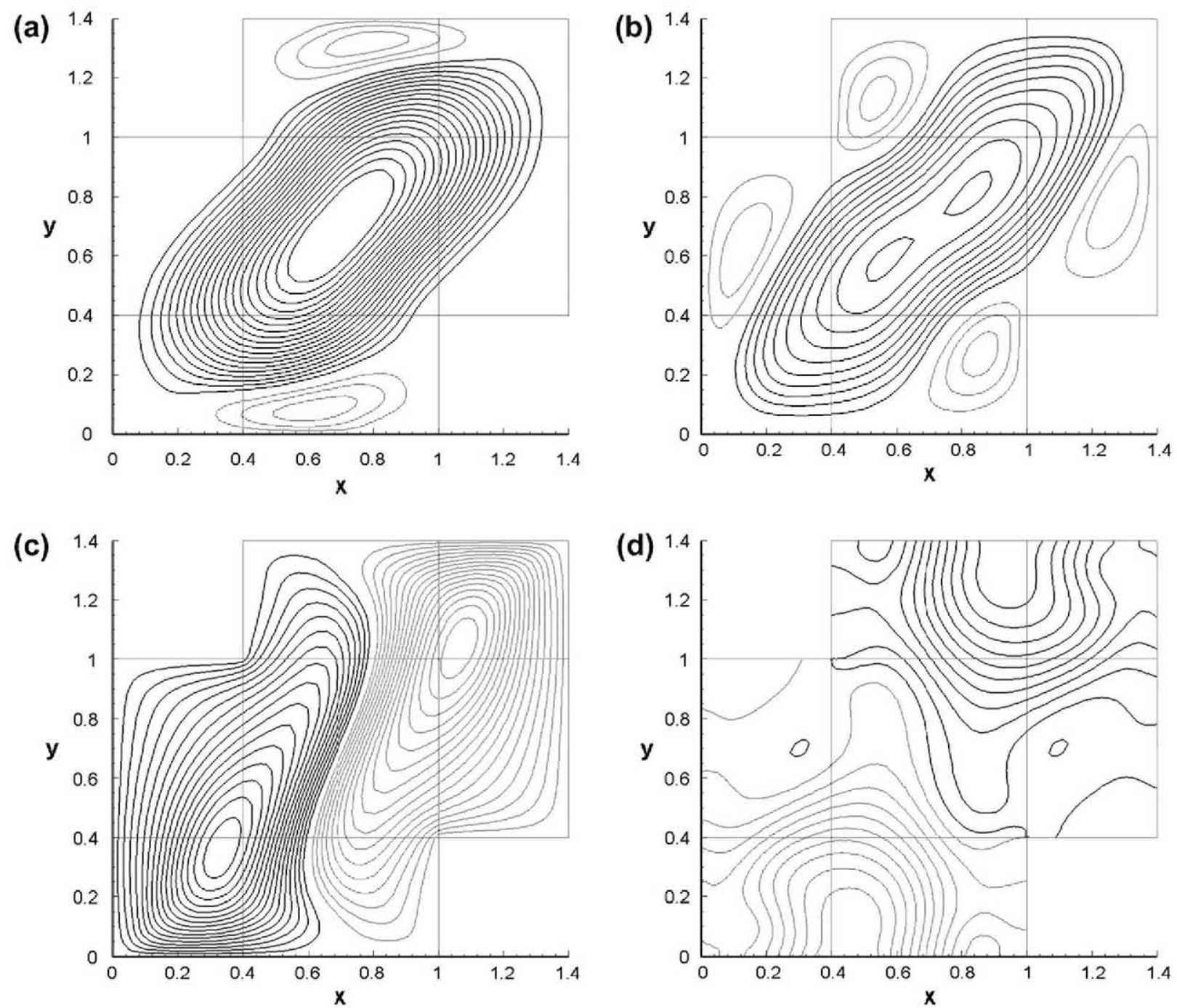

Fig. 7. Spatial structure of the non-dimensional amplitude functions of the disturbance velocity components and disturbance pressure of the leading eigenmode at $R e=170$, $\beta=2.42 ;\left(\omega_{r}=-0.2488 \times 10-3, \omega_{i}=0\right)$. (a) $(\hat{u}(x, y):(-0.15(0.05) 1) ;$ (b) $\hat{\nu}(x, y):(-0.15(0.05) 0.5) ;($ c $) \hat{w}(x, y):(-0.8(0.05) 0.8) ;(d) \hat{p}(x, y):(-1(0.1) 1)$.

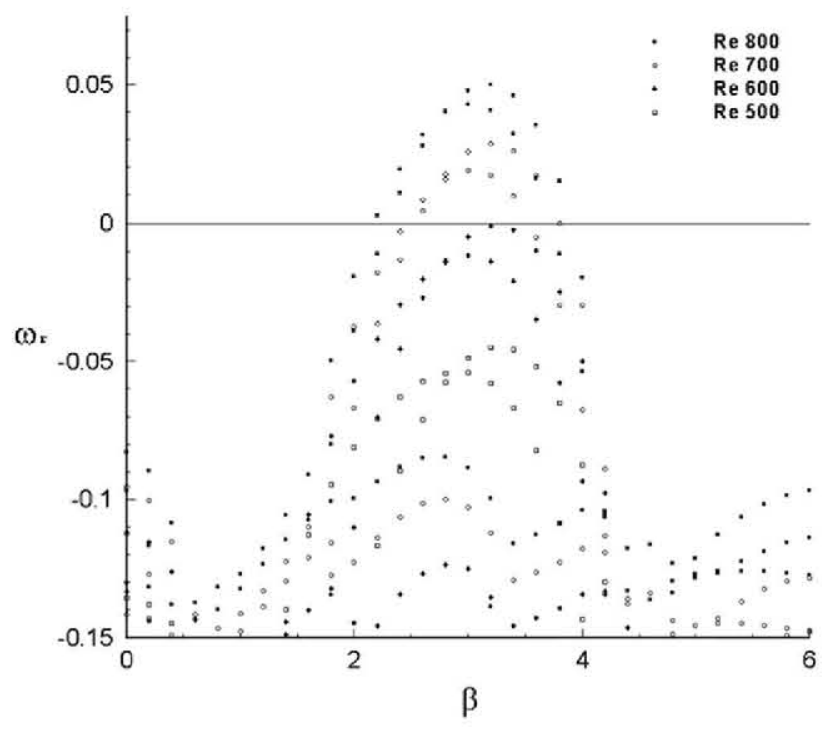

Fig. 8. Dependence of the amplification rate on the spanwise wavenumber parameter $\beta$ for a range of stable and unstable Reynolds numbers in the cross geometry.

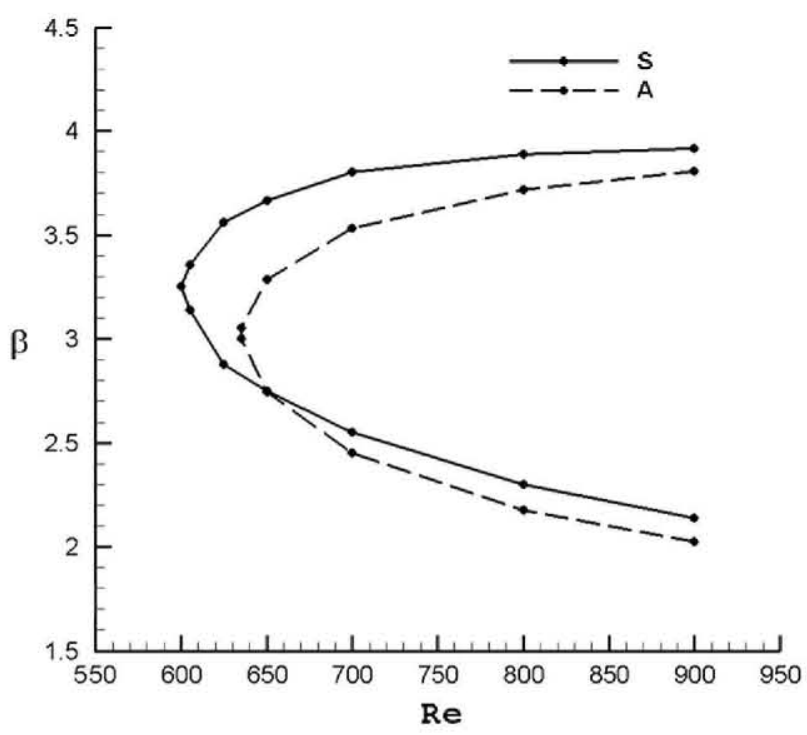

Fig. 9. Neutral stability curves of the symmetric, $S$, and anti-symmetric, $A$, modes in the cross-cavity: $R e_{\text {crit }, 3 \mathrm{~d}} \approx 602, \beta=3.25$. 
computations; as shown in Eq. (5), we have stopped the timeadvancement of the two-dimensional equations of motion when a relative discrepancy between successive time-steps is $O\left(10^{-14}\right)$, unlike both earlier analyses which utilized an absolute value for this quantity of $O\left(10^{-7}\right)$. Second, the present results have been obtained by spectral-multi-domain spatial discretization $[10,26]$, using at the highest resolution 40 collocation points per spatial direction in each subdomain; both of the earlier works used lower-order spatial discretization schemes. Finally, the regularization condition (6) is bound to generate minor but measurable differences compared with the singular boundary condition used in the previous works. Consequently, our computations deliver substantially more symmetric/anti-symmetric results than those of either of the previous two works, as would be expected from the geometric symmetry of the domain and boundary conditions. Table 3 tabulates vortex location and positions in the cross-cavity at $R e=200,500$ and 1000 .

Figs. 3 and 4 present basic flow streamlines and vorticity contours at several Reynolds numbers in the two geometries subsequently analyzed. Of interest here is the characteristic appearance and disappearance of the cats-eyes basic flow pattern, first seen in symmetrically-driven cavities in the work of Kuhlmann et al. [16]. Although direct comparisons are not possible in this context owing to the different geometries addressed, it is interesting to note that the cats-eyes pattern shown in Fig. 3 disappears at $R e_{\mathrm{z}} \approx 170$, while that in Fig. 4 disappears at $R e_{1} \approx 225$ and reappears at $R e_{2} \approx 600 ; R e_{2} \approx 170$ and $R e_{2} \approx 600$ have been found to correspond to the three-dimensional critical conditions for global instability in the respective geometries, as will be discussed shortly.

Instability analysis of symmetrically-driven cavity flows in the geometry defined by Hinatsu and Ferziger [15] proceeds along the usual lines of obtaining steady basic states at several Reynolds numbers, $R e$, and inquiring each of them with respect to its stability against three-dimensional perturbations of wavenumber, $\beta \in[0, \infty)$. In practice a large number of runs is performed at different $\beta$ values at each Reynolds number; the dependence of the amplification rate, $\omega_{r}$, on $\beta$, in a relatively coarse such scan of the parameter space is shown in Fig. 5. The results of significance here are, firstly, that two-dimensional $(\beta=0)$ global eigenmodes are more stable than their three-dimensional $(\beta \neq 0)$ counterparts, as well as that all basic states at $R e \geqslant 200$ are three-dimensionally unstable. In order to define the critical conditions in this geometry, the search of the parameter space has been refined, until the result shown in Fig. 6 was obtained. Here only results corresponding to the most unstable of the modes seen in Fig. 5 are shown; they have been used to define the neutral loop for this cavity geometry, also to be found in Fig. 6, without any cosmetic post-processing. The
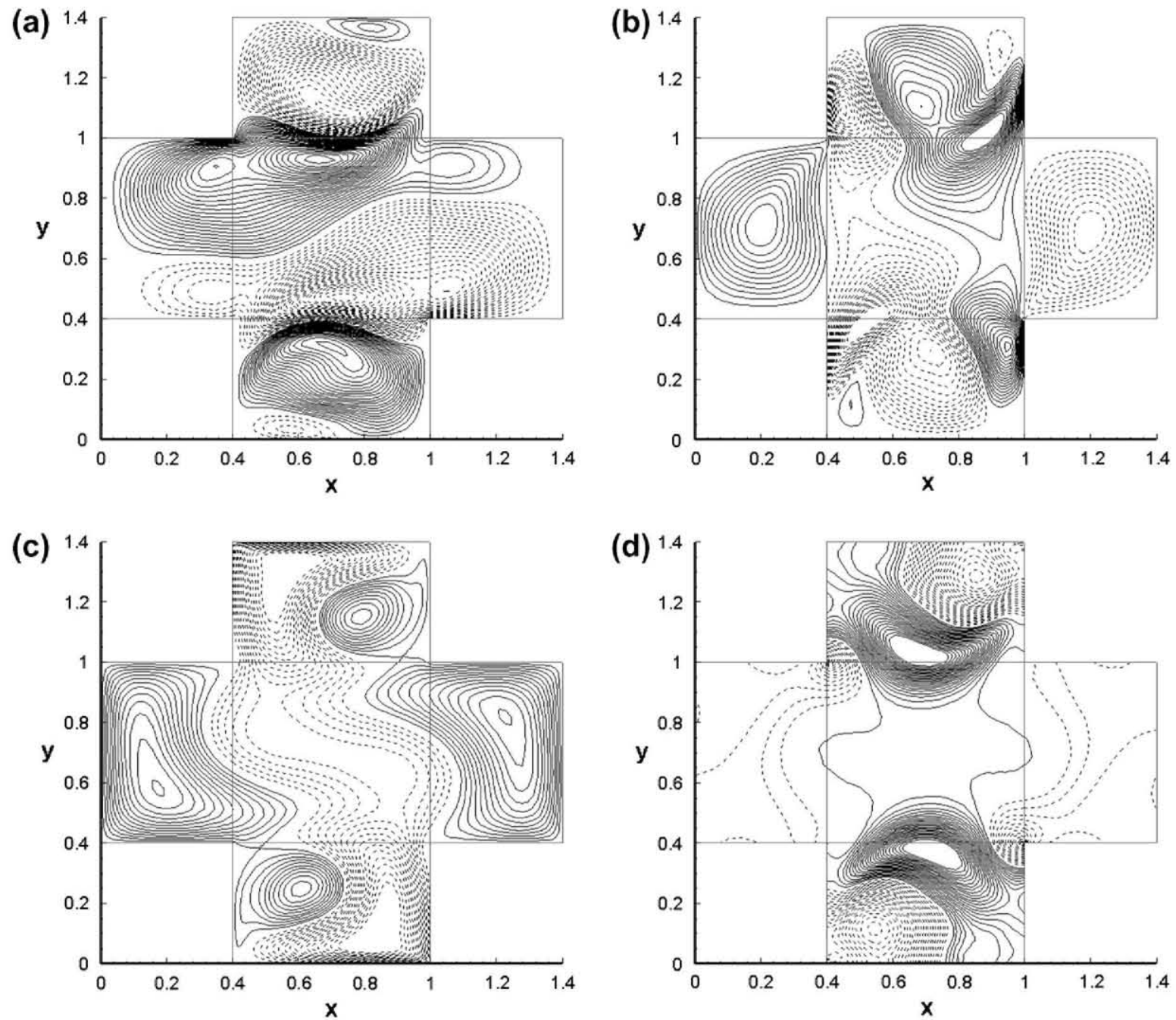

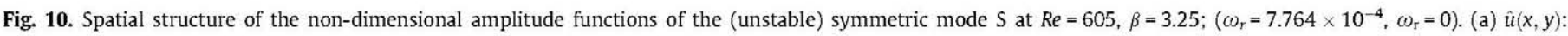
$-1(0.03) 1)$; (b) $\hat{v}(x, y)$ : $(-0.8(0.03) 0.8)$; (c) $\hat{w}(x, y):(-0.6(0.03) 0.6)$; (d) $\hat{p}(x, y):(-0.2(0.01) 0.2)$. 

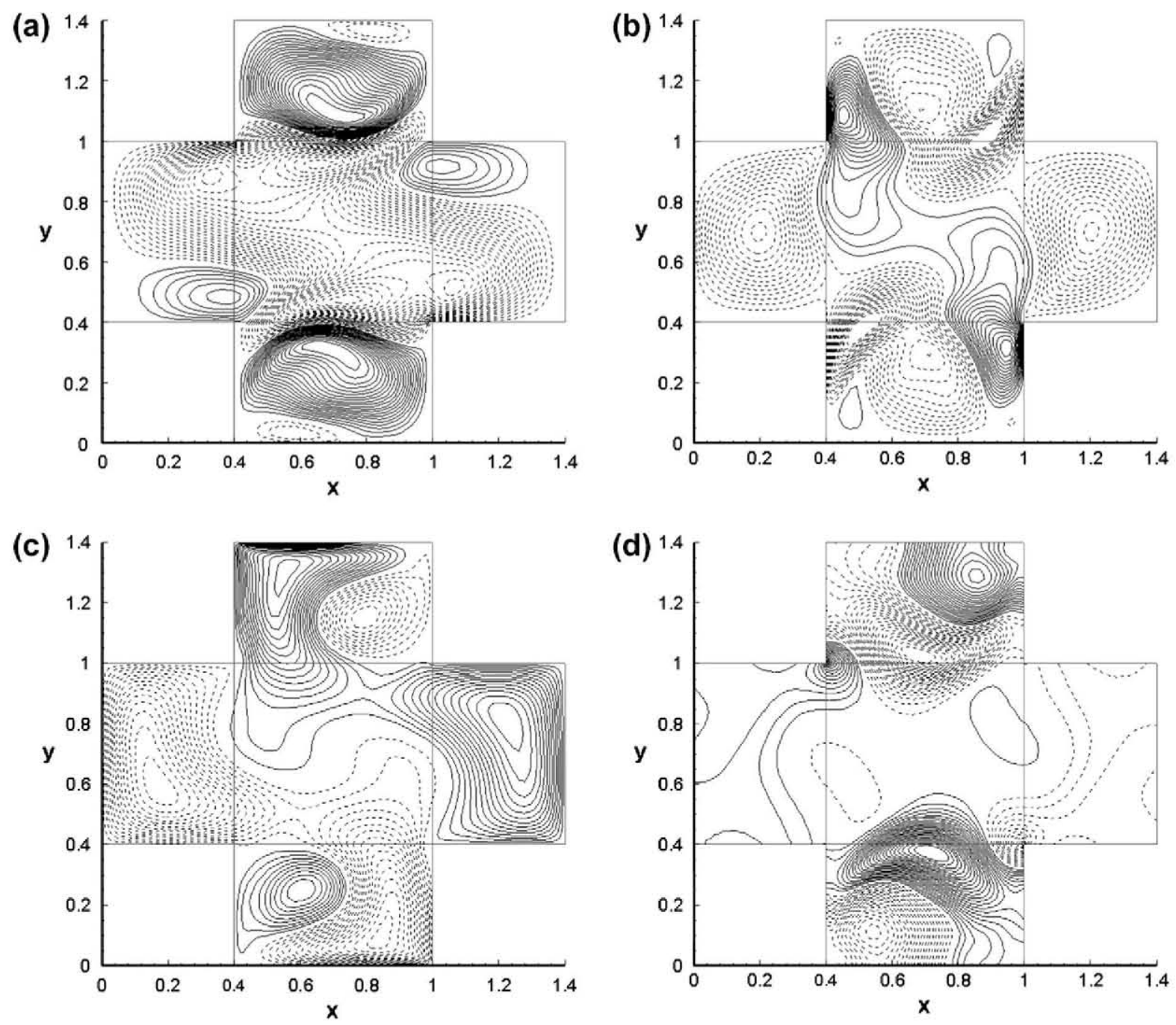

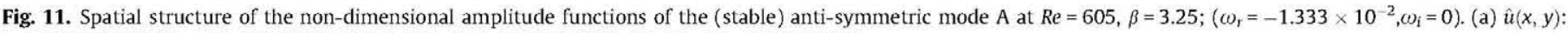
$(-1(0.03) 1)$; (b) $\hat{\nu}(x, y):(-0.8(0.03) 0.8) ;(c) \hat{w}(x, y):(-0.6(0.03) 0.6) ;(d) \hat{p}(x, y):(-0.2(0.01) 0.2)$.

amplitude functions of the leading eigenvector at (near) critical conditions are shown in Fig. 7. It is worth noting that the dominant perturbation has been found to be that corresponding to the streamwise disturbance velocity component, and the fact that the cats-eyes pattern of the (two-dimensional) basic state is modified into a structure related with this quantity at precisely the (three-dimensional) critical Reynolds number, $\operatorname{Re}_{\text {crit } 3 \mathrm{~d}} \approx 170$; the potential connections of these findings are presently explored, and will be reported elsewhere.

An analogous instability analysis in the cross-shaped symmetric-driven cavity geometry has delivered the amplification rate dependence on spanwise wavenumber shown in Fig. 8. Besides the rather larger Reynolds number for three-dimensional global instability, which can be asserted as $R e \geqslant 600$ on the basis of these results, another interesting difference with the results in the geometry analyzed previously is that two eigenmodes, becoming unstable at comparable conditions, have been identified in the cross-shaped cavity. Intuitively such a result may be expected on account of the point-symmetric nature of the geometry and boundary conditions in the cross-shaped cavity. A fine scan of the parameter space has delivered the neutral loops pertinent to these two modes, shown in Fig. 9. The critical conditions for this configuration were found to correspond to the symmetric mode, $\mathrm{S}$, as being $(\operatorname{Re} \approx 602, \beta \approx 3.25$ ). Interestingly, in this geometry too, $R e_{\text {crit } 3 \mathrm{~d}} \approx R e_{2}$ practically coincides with the reappearance of the cats-eyes pattern in the steady basic state. The amplitude functions of the leading symmetric and anti-symmetric eigenmodes are practically identical, up to a scaling factor, and are shown in Figs. 10 and 11 , respectively. However, small but perceptible differences have been found in the disturbance vorticity pattern pertinent to either mode, identified using the Q-criterion and seen in Fig. 12. In the same figure isosurfaces of the spanwise disturbance velocity component, $\hat{w}$, of the two modal disturbances can also be seen, making clear their symmetric/anti-symmetric nature. Such results should aid identification of the eigenmodes discovered herein in either three-dimensional direct numerical simulation or in experiments, both of which lie beyond the scope of the present analysis.

\section{Discussion}

The complexity of flow physics in lid-driven cavities helps discerning between algorithms capable of capturing such physics in an accurate manner. Although the particular features of the main and secondary vortices depend on the exact cavity geometry, the objective is always the exact determination of the location and strength of these vortices as a function of Reynolds number, as well as the calculation of the Reynolds number at which steady 
(a)

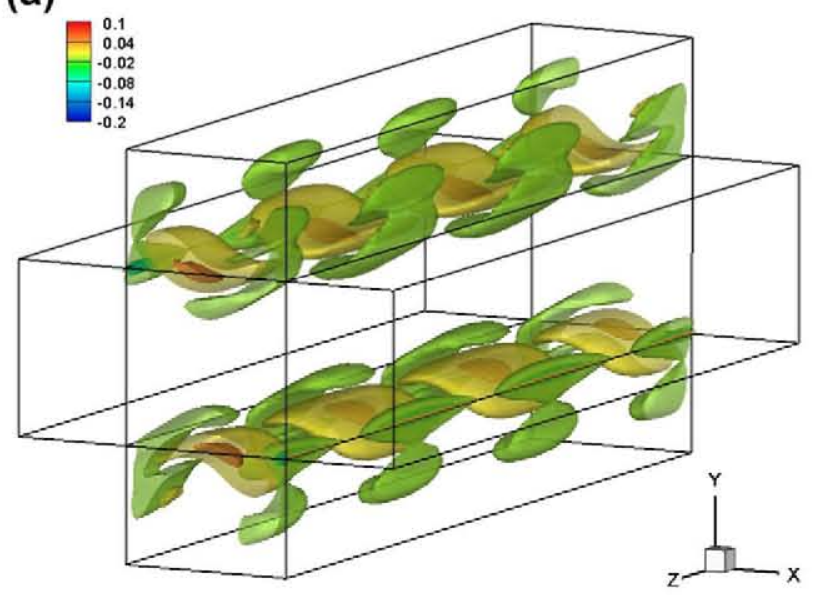

(c)
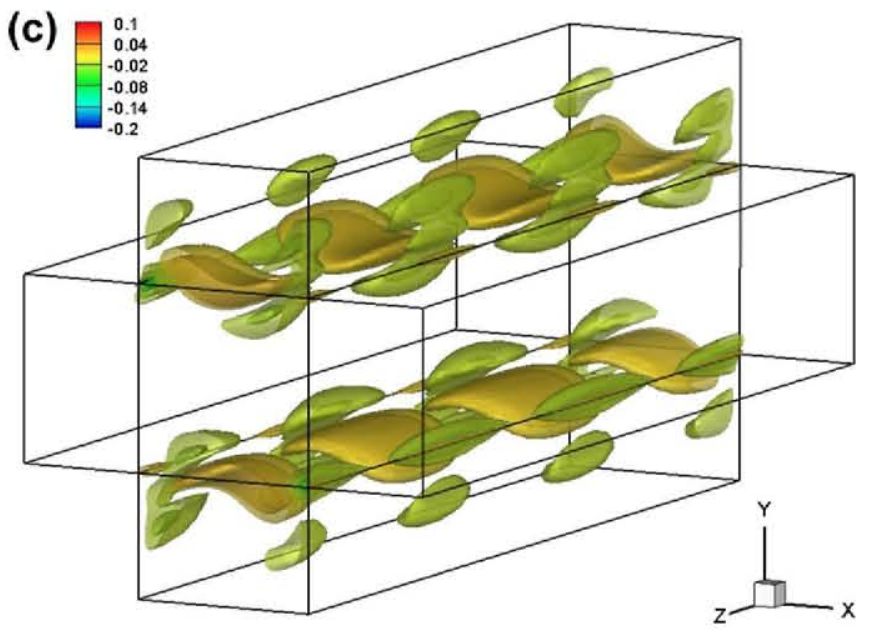

(b)

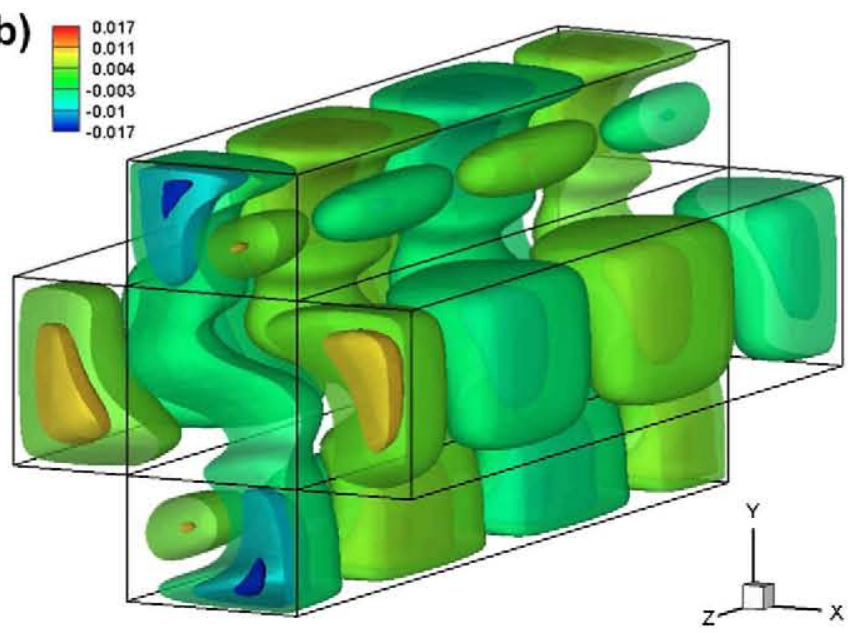

(d)

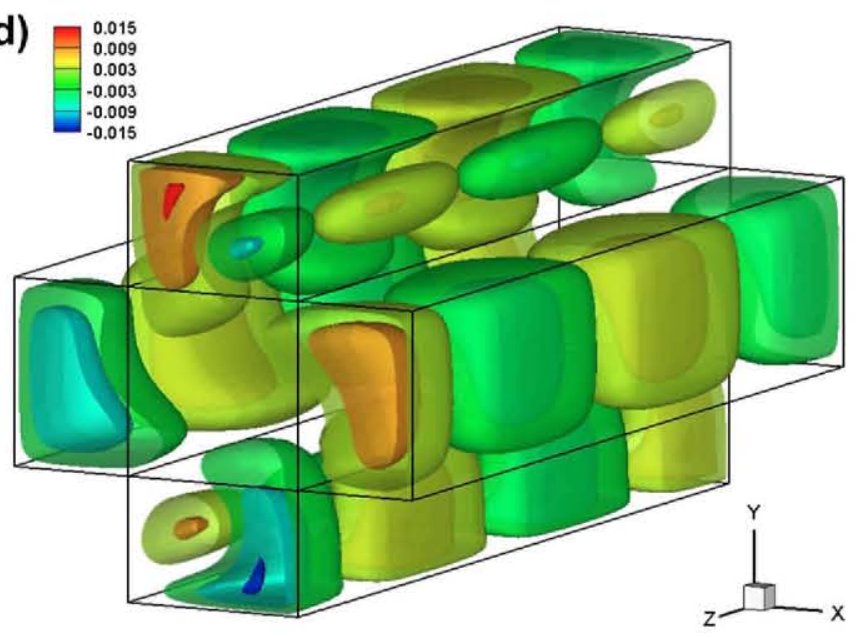

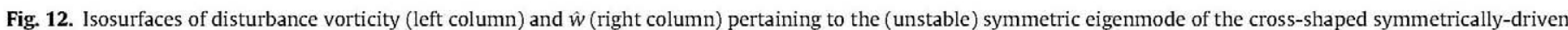
cavity (upper row) and the (stable) anti-symmetric eigenmode (lower row).

two-dimensional flow becomes unsteady. The differences between two- and three-dimensional lid-driven cavities have been documented numerically for decades. However, it was only in the last decade that global (BiGlobal) instability analysis [32] has been employed in order to explain such differences between spanwise homogeneous three-dimensional and purely two-dimensional flows $[30,2]$.

In the present work, two distinct algorithms for the solution of the BiGlobal eigenvalue problem, based on sparse serial and dense parallel technology, respectively, have been outlined and compared. Spectral collocation based on multi-domain discretization $[9,10,26]$ has been applied for spatial discretization of the eigenvalue problem in two basic flows analyzed, the point-symmetric geometry defined by Hinatsu and Ferziger [15] and a simpler point-symmetric version of that geometry. The sparse algorithm has been applied to analyze the two basic flows, define critical conditions and identify the leading eigenfunctions. While a single three-dimensional eigenmode was found in the first, two such eigendisturbances, one symmetric and one anti-symmetric were identified in the cross-cavity. The respective critical Reynolds numbers, $R e_{\text {crit,3d }} \approx 170$ and 602 , define the boundaries below which the numerically-obtained two-dimensional steady laminar solutions may be realized experimentally. Consequently, from a physical point of view, numerically-obtained solutions of the equations of motion above the respective $R e_{\text {crit,3d }}$ value can only be considered as numerically useful but physically unrealistic.

\section{Acknowledgments}

It is a pleasure to dedicate this paper to Michel Deville on the occasion of his $65^{\text {th }}$ birthday; his ground breaking contributions to the field of spectral-multidomain for the Navier-Stokes equations have formed the basis for the analysis presented herein. This work has been partially sponsored by the Air Force Office of Scientific Research, USAF, under grant number FA8655-03-1-3059 monitored by Dr. John D. Schmisseur (AFOSR) and Dr. Surya Surampudi (EOARD). The views and conclusions contained herein are those of the author and should not be interpreted as necessarily representing the official policies or endorsements, either expressed or implied, of the Air Force Office of Scientific Research or the US Government. Additional support has been offered by contract TRA/-2009-13648, Ministerio de Educación y Ciencia, Plan Nacional de Investigación.

\section{References}

[1] Ahusborde E Glockner S. A 2d block-structured mesh partitioner for accurate flow simulations on non-rectangular geometries. Comput. Fluids, (to appear), 2010.

[2] Albensoeder S, Kuhlmann HC, Rath HJ. Three-dimensional centrifugal-flow instabilities in the lid-driven-cavity problem. Phys Fluids $2001 ; 13(1): 121-36$.

[3] Arnoldi WE. The principle of minimized iterations in the solution of the matrix eigenvalue problem. Quart Appl Math 1951;9:17-29.

[4] Batchelor GK. On steady laminar flow with closed streamlines at large Reynolds number. J Fluid Mech 1956;1:177-90. 
[5] Burggraf OR. Analytical and numerical studies of the structure of steady separated flows. J Fluid Mech 1966;24:113-51.

[6] Chorin AJ. Numerical solution of the Navier-Stokes equations. Math Comp 1968;22:745-62.

[7] de Vicente J. Global instability analysis of complex cavity flows. PhD thesis. School of Aeronautics, Universidad Politécnica de Madrid; 2010.

[8] de Vicente J, Valero E, Gonzalez L, Theofilis V. Spectral multi-domain methods for bioglobal instability analysis of complex flows over open cavity configurations. AIAA-2006-2877 2006.

[9] Demaret P, Deville MO. Chebyshev pseudospectral solution of the Stokes equations using finite element preconditioning. J Comp Phys 1989;83(2):463-84

[10] Demaret P, Deville MO. Chebyshev collocation solutions of the Navier-Stokes equations using multi-domain decomposition and finite element preconditioning. J Comp Phys 1991;95(2):359-86.

[11] Deville MO, Fischer PF, Mund EH. High order methods for incompressible fluid flow. Cambridge University Press; 2002.

[12] Ding Y, Kawahara M. Linear stability of incompressible flow using a mixed finite element method. J Comp Phys 1998;139:243-73.

[13] Erturk E. Discussions on driven cavity flow. Int J Numer Meth Fluids $2009 ; 60: 275-94$.

[14] Ghia U, Ghia KN, Shin CT. High-Re solutions for incompressible flow using the Navier-Stokes equations and a multigrid method. J Comp Phys 1982;48(3):387-411.

[15] Hinatsu M, Ferziger JH. Numerical computation of unsteady incompressible flow in complex geometry using a composite multigrid technique. Int J Numer Meth Fluids 1991;13(8):971-97.

[16] Kuhlmann HC, Wanschura M, Rath HJ. Flow in two-sided lid-driven cavities: non-uniqueness instabilities and cellular structures. J Fluid Mech 1997;336:267-99.

[17] Lehoucq Richard B, Sorensen Danny C, Yang C. Arpack user's guide: solution of large-scale eigenvalue problems with implicitly restarted Arnoldi methods (software, environments, tools). Soc Indus \& Appl Math 1997.

[18] Leriche E, Gavrilakis S, Deville MO. Direct simulation of the lid-driven cavity flow with chebyshev polynomials. In: Papailiou KD, editor, Proc. 4 th european computational fluid dynamics conference, vol. 1; 1998. p. 220-5.
[19] Li XS, Demmel JW. SuperLU ${ }_{\text {DIST }}$ a scalable distributed-memory sparse direct solver for unsymmetric linear systems. ACM Trans Math Software 2003;29(2):110-40.

[20] Moffatt HK. Viscous and resistive eddies near a sharp corner. J Fluid Mech 1964;18:1-18.

[21] Nithiarasu P, Liu C-B. Steady and unsteady incompressible flow in a double driven cavity using the artificial compressibility (AC)-based characteristic-based split (CBS) scheme. Int J Numer Meth Eng 2005;63:380-97.

[22] Oosterlee CW, Wesseling P, Segal A, Brakkee E. Benchmark solutions for the incompressible Navier-Stokes equations in general coordinates on staggered grids. Int J Numer Meth Fluids 1993;17:301-21.

[23] Pan F, Acrivos A. Steady flows in rectangular cavities. J Fluid Mech 1967;28:643-55.

[24] Rodríguez D, Theofilis V. Massively parallel numerical solution of the BiGlobal linear instability eigenvalue problem using dense linear algebra. AIAA J 2009;47(10):2449-59.

[25] Saad Y. Variations of Arnoldi's method for computing eigenelements of large unsymmetric matrices. Lin Algebra Appl 1980;34:269-95.

[26] Schneidesch CR, Deville MO. Chebyshev collocation method and multi-domain decomposition for Navier-Stokes equations in complex curved geometries. J Comp Phys 1993;106:234-57.

[27] Schreiber R, Keller HB. Driven cavity flows by efficient numerical techniques. J Comp Phys 1983;49(2):310-33.

[28] Shankar PN, Deshpande MD. Fluid mechanics in the driven cavity. Annu Rev Fluid Mech 2000;32:93-136.

[29] Temam R. Navier-Stokes equations and nonlinear functional analysis. Philadelphia: SIAM; 1995.

[30] Theofilis V. Globally-unstable flows in open cavities. AIAA Paper 2000-1965 2000.

[31] Theofilis V. Advances in global linear instability of nonparallel and threedimensional flows. Prog Aero Sci 2003;39(4):249-315.

[32] Theofilis V. Global linear instability. Annu Rev Fluid Mech 2011;43: 319-52.

[33] Zhou YC, Patnaik BSV, Wan DC, Wei GW. Dsc solution for flow in a staggered double lid driven cavity. Int J Numer Meth Eng 2003;57:211-34. 